\title{
Nutrizione iodica in gravidanza e sviluppo cerebrale: associazione tra ioduria materna e morfologia cerebrale della progenie
}

\author{
Mariacarla Moleti $^{1} \cdot$ Maria Di Mauro ${ }^{1}$. Francesco Vermiglio ${ }^{1}$
}

Accettato: 14 gennaio 2022 / Pubblicato online: 21 gennaio 2022

(c) The Author(s), under exclusive licence to Springer Nature Switzerland AG 2022

Commento a:

Urinary iodine concentrations in pregnant women and offspring brain morphology.

T.A. Mulder, T.I. Korevaar, R.P. Peeters, A.E. van Herwaarden, Y.B. de Rijke,

T. White, $\mathrm{H}$. Tiemeier.

Thyroid (2021) 31(6):964-972

Recenti stime dello Iodine Global Network indicano come, ancora oggi, in circa 1/5 dei paesi al mondo l'apporto nutrizionale di iodio nelle donne in gravidanza risulti lievementemoderatamente inadeguato a soddisfare il fabbisogno gestazionale di tale micronutriente. Una simile condizione carenziale è stata variabilmente associata a compromissione dello sviluppo neuro-intellettivo della progenie: accanto a studi che indicano una significativa correlazione tra iodocarenza materna e alterazioni cognitive dei nati, ve ne sono altri dai quali tale relazione non risulta evidente [1]. La contraddittorietà di tali risultati è attribuibile - almeno in parte - a differenze nella metodologia (modalità e tempistica di determinazione dello stato di nutrizione iodica, selezione dei gruppi di controllo e/o dei fattori confondenti), nell'età dei bambini al momento della valutazione neuro-cognitiva e, non ultimo, nello stato di nutrizione iodica delle donne prima del concepimento [2]. I risultati della ricerca di Mulder e collaboratori forniscono un'ulteriore possibile chiave di lettura delle attuali evidenze scientifiche, in quanto indicano come anche l'esposizione fetale all'eccesso di iodio si associ ad alterazioni morfologiche cerebrali rilevabili in epoca post-natale.

Realizzato nell'ambito del progetto Generation $R$, questo studio si prefiggeva di esplorare l'associazione tra escrezione urinaria di iodio delle madri durante la gravidanza e

\footnotetext{
M. Moleti

mariacarla.moleti@unime.it

1 Dipartimento di Medicina Clinica e Sperimentale, Università degli Studi di Messina, Messina, Italia
}

morfologia cerebrale dei nati, quest'ultima valutata in età preadolescenziale mediante imaging a risonanza magnetica (RMI). Lo studio ha coinvolto una casistica di 990 coppie madri-bambini e ha previsto la misurazione della ioduria materna (espressa come rapporto UI/Creat) in differenti epoche gestazionali $(<18,18-25$ e $>25$ settimane di gravidanza) e l'esecuzione della MRI cerebrale nei nati all'età di 10 anni. Benché la ioduria media nell'intera popolazione studiata indicasse una condizione di iodo-sufficienza (UI/Creat $224 \mu \mathrm{g} / \mathrm{g}$ ), nel 20\% delle donne l'escrezione urinaria di iodio (valutata come media tra le diverse determinazioni) si collocava nel range della carenza (UI/Creat $<150 \mu \mathrm{g} / \mathrm{g}$ ) e in una percentuale non precisata in quello dell'eccesso (UI/Creat 250-499 $\mu \mathrm{g} / \mathrm{g}$ ). L'analisi di regressione adattata (spline regression) dimostrava come la relazione tra ioduria materna e le diverse misure di outcome morfologico alla MRI non fosse lineare bensì curvilinea, e tale che valori di UIC sia bassi che alti si associassero a volumi di materia grigia nei nati significativamente ridotti.

Se confermati, questi risultati suggeriscono che, come la iodocarenza, anche l'eccesso di iodio può compromettere lo sviluppo cerebrale e che l'apporto gestazionale di questo nutriente deve essere mantenuto entro limiti adeguatamente definiti.

\section{Bibliografia}

1. Lee SY (2021) Editorial: consequences of iodine deficiency in pregnancy. Front Endocrinol 12:740239

2. Levie D, Korevaar TIM, Bath SC et al (2019) Association of maternal iodine status with child IQ: a meta-analysis of individual participant data. J Clin Endocrinol Metab 104(12):5957-5967

Nota della casa editrice Springer Nature rimane neutrale in riguardo alle rivendicazioni giurisdizionali nelle mappe pubblicate e nelle affiliazioni istituzionali. 